\title{
Title: The need to redefine age- and gender-specific overweight and obese body mass index (BMI) cut-off points.
}

Authors: Alan. M. Nevill, $\mathrm{PhD}^{1,3}$, George S. Metsios $\mathrm{PhD}^{1,2,3}$,

${ }^{1}$ Research Institute of Healthcare Sciences, University of Wolverhampton, Walsall, UK.

${ }^{2}$ Department of Rheumatology, Dudley Group of Hospitals NHS Trust, Russell’s Hall

Hospital, Dudley, West Midlands, UK.

${ }^{3}$ Research Institute in Physical Performance and Rehabilitation, Centre for Research and Technology - Thessaly, Trikala, Greece.

Address for correspondence:

Professor Alan M. Nevill, Ph.D.

University of Wolverhampton

Research Institute of Healthcare Sciences, Walsall Campus

Gorway Road

Walsall, WS1 3BD

Tel: +44 (0)1902 322838

Fax: +44 (0)1902 322894

Email: a.m.nevill@wlv.ac.uk 


\section{Abstract}

For convenience, health practitioners and clinicians are inclined to classify people/patients as overweight or obese based on body mass index (BMI) cut-off points of 25 and $30 \mathrm{~kg} / \mathrm{m}^{2}$, respectively, irrespective of age and gender. The purpose of the current study was to identity whether, for the same levels of adiposity, BMI is the same across different age groups and gender. A two-way ANCOVA revealed significant differences in BMI between different age groups and gender (plus an interaction), using body fat (\%) as the covariate, data taken from a random sample of the English population ( $\mathrm{n}=2993)$. Younger people had greater BMI than older people for the same levels of adiposity (differences ranged by 4 BMI units for males, and 3 BMI units for females). In conclusion, if BMI thresholds for overweight (BMI=25 kg/m²) and obese $\left(\mathrm{BMI}=30 \mathrm{~kg} / \mathrm{m}^{2}\right)$ are to reflect the same levels of adiposity across all gender and age groups within a population, age- and gender-specific BMI adjustments outlined here are necessary to more accurately/fairly reflect the same critical levels of adiposity.

Keywords: body mass index, obesity, body fat, epidemiology, analysis of covariance 


\section{Introduction}

Body mass index (BMI) is undoubtedly the most frequently used proxy of adiposity/obesity in large epidemiological studies in both healthy and diseased populations. Despite its wide use, which pertains to convenience since it only requires the measurement of height and mass, BMI has been frequently criticised as having various deficiencies as a measure of obesity [1] both for healthy and diseased populations [2, 3].

One of the major issues with BMI is that it does not reflect the changes in body composition that occur with age, in particular the presence of sarcopaenia which is characterised by reduced muscle mass and increased adiposity. As such the utilisation of BMI in evidencebased approaches relevant to dietary interventions and/or clinical decision making needs to be reconsidered and where appropriate, re-adjusted. Therefore, the aim of the present study was to investigate the cut-off points of BMI in relation to adiposity in a large cohort of participants in order to validate if the established cut-off points accurately reflect adiposity.

\section{Methods}

The current data, used to explore the association between BMI $\left(\mathrm{kg}^{\left.-\mathrm{m}^{-2}\right)}\right.$ and body fat percentage (BF\%), has been previously published [4] although originally obtained from the Allied Dunbar National Fitness Survey (ADNFS) (1992). The ADNFS recruited 4316 randomly selected healthy participants, aged 16 years and over, from thirty English parliamentary constituencies. A sub-sample took part in a physical appraisal yielding BMI and estimates of BF\% data for 2993 healthy people (male $n=1420$; female $n=1573$ ). Estimates of BF\%, taken for the ADNFS, were determined using the methods based on skin fold thicknesses at four sites; the biceps, triceps, sub-scapular and supra-iliac [5].

\section{Statistical Methods}

In order to detect any systematic differences in gender and age groups (16-29, 30-39, 40-49, 50-59, 60-69, 70-79, 80+, age in years) holding BF\% constant, a two way (gender-by-age 
group) analysis of covariance (ANCOVA) was employed using BF\% as the co-variate. Finally, we have used Bonferroni multiple comparisons to investigate BMI differences amongst the different age groups. The level of significance was set at $\mathrm{P}<0.05$ and all the analyses were conducted with the Statistical Package for the Social Sciences (SPSS) version 20.

\section{Results}

The relationship between BMI and BF\% was found to be approximately linear. The ANCOVA revealed significant main effects of age group $(\mathrm{P}<0.001)$ and gender $(\mathrm{P}<0.001)$, and a significant age group-by-gender interaction $(\mathrm{P}<0.001)$ together with a significant covariate of $\mathrm{BF} \%$ with the $\mathrm{BMI}$ slope parameter $\mathrm{B}=0.570$ ( $\mathrm{SE}=0.010)$ per unit $\mathrm{BF} \%$, means $( \pm \mathrm{SE})$ given in Figure 1. The group-by-gender interaction was due to a greater difference in BMI between the younger and older males compared with the difference in BMI observed in females.

Figure 1 about here

The median age of the sample was 45 years. As such, taking the "anchored” baseline group as the 40-49 year old group, and assuming the overweight and obese threshold for these groups are $\mathrm{BMI}=25$ and $30 \mathrm{~kg} / \mathrm{m}^{2}$ respectively, we estimated the BMI of all other age groups and by gender, based on the differences observed in Figure 1. Anchoring the overweight threshold BMI value $=25 \mathrm{~kg} / \mathrm{m}^{2}$ for the $40-49$ age group, all other age group and gender differences are estimated in Table 1 [BMI means (rounded to a whole BMI unit number) estimated for the same BF\% = 25.3 and 34.8 for all male and female participants, respectively]. Similarly anchoring the obese threshold BMI value $=30 \mathrm{~kg} / \mathrm{m}^{2}$ for the $40-49$ age group, all other age group and gender differences are estimated in Table 1 [BMI means (rounded) estimated for the same $\mathrm{BF} \%=34.1$ and 43.5 for all male and female participants, respectively]. 
Table 1 about here

\section{Discussion}

For the same level of adiposity (using BF\%), systematic differences in BMI were found in different gender and age groups from a randomly selected national sample (male n=1420; female $n=1573$ ) taken from 30 English parliamentary constituencies (see Figure 1). Based on these differences, the BMI means (rounded), calculated and reported in Table 1 suggest that younger males and, to a lesser extent female participants have significantly higher levels of BMI compared with their older counterparts, for the same levels of adiposity (BF\%). This is unsurprising given that younger males are likely to be more active than younger females and that physical activity will naturally decline in both genders in older people. This trend is well documented with ageing [6], in particular the presence of sarcopaenia that is characterised by reduced muscle mass and increased adiposity, the latter being the result of lower energy expenditure.

Although this trend is well known amongst health practitioners, most still persist in prescribing common BMI thresholds for being overweight $\left(\mathrm{BMI}=25 \mathrm{~kg} / \mathrm{m}^{2}\right)$ and obese $\left(\mathrm{BMI}=30 \mathrm{~kg} / \mathrm{m}^{2}\right)$ irrespective of the individuals age and gender. Our findings suggest an alternative strategy should be considered. Given that younger males will have a higher percentage of muscle mass than a 40-49 year old male, their BMI obesity threshold could be raised to a less restrictive $33 \mathrm{~kg} / \mathrm{m}^{2}$ (as per our relevant calculations in Table 1) to equate to the same level of adiposity as their older 40-49 year old counterparts. A similar recommendation can be made for younger females, i.e. their BMI obesity threshold could be raised to $32 \mathrm{~kg} / \mathrm{m}^{2}$ (please see Table 1 a for newly developed cut-off points) to equate to the same level of adiposity as a 40-49 year old female. In contrast, the BMI obesity threshold for 
both male and female 50 to 59 year old participants could be reduced to a more restrictive/conservative level. For these older age groups, we would recommend that such participants are regarded as obese once their BMI exceeds 29 rather than $30 \mathrm{~kg} / \mathrm{m}^{2}$ (to equate with the same level of adiposity associated with 40-49 year old subjects). Similar adjustments would be required for the overweight thresholds for the various gender and age groups outlined in Table 1.

In conclusion, if BMI thresholds for overweight and obese participants are to reflect similar levels of adiposity across all gender and age groups within a population, age and gender specific BMI adjustments outlined in Table 1 are necessary to more accurately and fairly reflect the same levels of adiposity.

\section{Conflict of Interest statement}

The authors declare no conflict of interest. 


\section{References}

1. Rothman, K.J., BMI-related errors in the measurement of obesity. Int J Obes (Lond), 2008. 32 Suppl 3: p. S56-9.

2. Nevill, .A.M, Stavropoulos-Kalinoglou A., Metsios G.S., Koutedakis Y., Holder R.L., Kitas G.D., et al., Inverted BMI rather than BMI is a better proxy for percentage of body fat. Ann Hum Biol, 2011. 38(6): p. 681-4.

3. Stavropoulos-Kalinoglou A, Metsios GS, Yiannis Koutedakis Y, Nevill AM, Douglas KM, Jamurtas A, et al., Redefining overweight and obesity in rheumatoid arthritis patients. Ann Rheum Dis, 2007. 66(10): p. 1316-21.

4. Nevill, A.M. and R.L. Holder, Body mass index: a measure of fatness or leanness? $\mathrm{Br}$ J Nutr, 1995. 73(4): p. 507-16.

5. Durnin, J.V. and J. Womersley, Body fat assessed from total body density and its estimation from skinfold thickness: measurements on 481 men and women aged from 16 to 72 years. Br J Nutr, 1974. 32(1): p. 77-97.

6. Fragala, M.S., A.M. Kenny, and G.A. Kuchel, Muscle quality in aging: a multidimensional approach to muscle functioning with applications for treatment. Sports Med, 2015. 45(5): p. 641-58.

\section{Legend to Figures}

Figure 1 Mean $( \pm$ SE) BMI by age-group and by gender holding BF\% constant, all BMIs evaluated at $\mathrm{BF} \%=29.49 \%$. 